\title{
ETAT DE DROIT ET TERRORISME EN AFRIQUE CENTRALE : QUELS ENJEUX POUR LA REPUBLIQUE DEMOCRATIQUE DU CONGO?
}

\author{
Par Mö̈se ABDOU MUHIMA ${ }^{1}$ \\ “C'est lorsqu'on est environné de tous les dangers qu'il n'en faut redouter aucun ${ }^{2} »$
}

\begin{abstract}
Résumé
Cette étude est une sonnette d'alarme destinée aux dirigeants politiques de la République Démocratique du Congo, pour qu'ils perçoivent la nécessité et les enjeux de la prévention et de la répression du terrorisme en $\mathrm{RDC}$, qui constitue un enjeu majeur dans la géostratégie et la géopolitique de la recrudescence de l'extrémisme en Afrique et dans la région, menaçant la nature même de l'Etat de droit. C'est pourquoi, la RDC devrait être la première nation à prendre des précautions de haute facture pour lutte contre ce fléau, car de sa stabilité, dépend celle de ces neuf voisins, repartis sur trois régions africaines. Le danger est la dynamique de l'exportation de la menace des Shebaab somaliens qui combattent aux côtés des groupes rebelles qui sévissent dans de vastes régions forestières au Nord-est de la RDC, auxquels il faut ajouter la vague de l'Etat islamique en Afrique de l'Ouest, ex-Boko haram, qui hante l'Afrique de l'Ouest sans épargner l'Afrique centrale. Cette dynamique menace l'Etat dans son existence effective; et le désastre pour la région et pour la RDC reste par ailleurs l'inadéquation du système congolais de prévention, de lutte et de pression de l'extrémisme violent et du terrorisme qui n'est constitué que d'un arsenal juridique globalisant et d'institutions techniques spécialisées qui ne tiennent pas compte des particularités, des enjeux et des intérêts africains et congolais en présence.
\end{abstract}

Mots clés : Afrique centrale - RDC - Etat de droit -terrorisme -menace - lutte - répression.

\footnotetext{
Abstract

This study is a wake-up call for the political leaders of the Democratic Republic of Congo, for they see the need and challenges of prevention and suppression of terrorism in the DRC which is a major issue in the geostrategy and geopolitics of the upsurge of extremism in Africa and in the region threatening the contemporary nature of the rule of law. That is why

1 Doctorant en droit à l'Université de Kinshasa (RDC) / Membre du CODESRIA (Conseil pour le développement de la recherche en sciences sociales en Afrique, Dakar/Sénégal)

E-mail : muhimamoise@gmail.com. Téléphone+243810585853.

2 COUTTE A., Irak. La ruée vers l'or, France, 2003, p.251.
} 
it should be the first nation to take precautions high bill for fight against this scourge, because of its stability, depends on which of these nine neighbors spread over three African regions. The danger is the dynamics of the export of the threat of Somali Shebaab fighting alongside rebel groups operating large forest areas in north-eastern $\mathrm{DRC}$, to which must be added the wave of the Islamic state in West Africa, former Boko haram, which haunts the West African sparing Central Africa. This dynamic threatens the state in its contemporary existence; and the disaster for the region and for the DRC also remains inadequate Congolese system of prevention, control and pressure of violent extremism and terrorism which consists only of a legal arsenal globalizing and specialized technical institutions that do not take into account the specificities, challenges and interests of African and Congolese involved.

Keywords: Central Africa - DRC - Rule of Law - terrorism - threat- fight- suppression.

\section{Introduction}

L'Etat de droit serait comparable à ce Gouvernement des lois, pas des hommes, et pourtant, où sévit le terrorisme, il n'y a point de lois, seul la terreur règne en "Maitre incontesté ». L'Etat de droit exige aux autorités politiques et administratives de se soumettre aux règles de droit ${ }^{3}$, et l'accent est mis sur le respect des principes et valeurs fondamentaux, notamment les droits de l'homme, les libertés publiques et la séparation des pouvoirs ${ }^{4}$. Cela supposant notamment l'indépendance de la justice qui doit assurer le respect de lois en toute

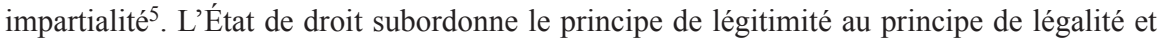
prohibe l'arbitraire ${ }^{6}$. Dans ce contexte, aucun individu, aucune institution n'est au dessus de la loi et ici, la terreur n'a aucune raison d'être vécue ${ }^{7}$, bien entendu, il importe d'agir efficacement dans ce contexte sans trahir les valeurs de la démocratie ${ }^{8}$.

A ce jour, l'Afrique centrale est le théâtre de deux courants d'actes barbares et scandaleusement dangereux au principe de la démocratie, de l'Etat de droit et au respect des droits

3 CEDRONIO M., Hannah Arendt : Politique et histoire. La démocratie en danger, Paris, 1999, p.11.

4 NTUMBA-LUABA LUMU D.A., Droit constitutionnel général, Kinshasa, éditions universitaires africaines, 2007, pp. 57-58.

5 MBATA B. MANGU A., Abolition de la peine de mort et constitutionnalisme en Afrique, Paris, 2011, p.34.

6 CARLIER J.-Y., « De l'Etat de droit à l'état des droits », Journal des procès, n²13, 20 mars 1992, pp. 32-44.

7 BAYART J. F., « Le crime transnational et la formation de l'État », Politique africaine $\mathrm{n}^{\circ} 93$ - mars 2004, pp. 93-104.

8 CERDA-GUZMAN Carolina, « La Constitution : une arme efficace dans le cadre de la lutte contre le terrorisme? », Revue française de droit constitutionnel, 2008/1 (n 73$)$, p. 41-63. 
et libertés fondamentaux. Le premier représente ce qu'il faut appeler le «terrorisme d'Etat $^{9}$ », actes odieux commandités par les individus détenteurs du pouvoir politique dans les Etats de la région. Et certains auteurs soulignent notamment qu'en RDC, les violations répétitives de la loi par le Gouvernement constituent la forme la plus visible et la plus courante de violation des principes de $1^{\prime}$ Etat de droit ${ }^{10}$. Le deuxième courant est quant à lui orchestré par des groupes rebelles qui profitent de la « faillite de l'Etat $»^{11}$, pour semer la terreur en Afrique centrale.

En fait, faudrait-il révéler ici qu'il y a un nombre considérable de Conventions et Protocoles internationaux qui s'intéressent directement ou indirectement à cette seconde forme de barbarie qualifiée de terrorisme ${ }^{12}$. Le constat est que chacun de ces instruments porte, pour la plupart, sur des comportements criminels spécifiques et moins sur la notion plus générale de «terrorisme » en tant que tel. Malgré l'existence de plus d'une douzaine de Conventions Internationales contre le terrorisme, il n'existe pas de définition unanime de la notion de «terrorisme», car les Etats et les opinions continuent à diverger au sujet de cette définition ${ }^{13}$. Mais faudrait-il noter que certains instruments juridiques internationaux donnent une définition de la notion de «terrorisme ", de ce nombre figure la Convention arabe contre le terrorisme qui prévoit à son article $1^{\text {er }}$ (2) que :

"le Terrorisme s'entend de tout acte ou menace de violence, quels que soient ses motifs ou les buts, qui serait l'instrument d'un projet criminel individuel ou collectif, et viserait à semer la terreur dans la population, à lui inspirer la peur, en lui portant préjudice ou en mettant sa vie, sa liberté ou son indépendance en péril, à causer des dommages à l'environnement, à une installation ou à un bien, tant public que privé, à

9 CAMUS A., L'homme révolté, Paris, 1951, p. 221; BERNARD HUYGHE (F.), Anthologie de textes sur la polémologie, Une science de la guerre?, Paris, 2008, p.21; CAMPANA (A.) et HERVOUET (G.) dir., Terrorisme et insurrection : évolution des dynamiques conflictuelles et réponses des Etats, Québec, 2013, pp. 8-14; MONDO B., « Même les monarques absolus du XVII ${ }^{\text {ème }}$ siècle ne se comportent pas de cette façon avec leurs citoyens ", disponible sur www.jeunetchad.mondoblog.org., publié le 19 août, 2014.

10 KIFWABALA TEKILAZAYA, FATAKI wa LUHINDI D., WETSH'OKONDA KOSO M., République démocratique du Congo. Le secteur de la justice et l'Etat de droit, Johannesburg, 2013, p. 33.

11 NZONGOLA-NTALAJA G., " The fragile State and crisis in eastern Congo ", African Journal of democraty and governance (AJDG), Vol. 2, n $1 \& 2$, Kinshasa, 2015, pp. 15-37, DEVLIN-FOLTZ (Z.), « Les États fragiles de l'Afrique : vecteurs de l'extrémisme, exportateurs du terrorisme », Bulletin de la sécurité africaine, $n^{\circ}$ 6, Washington DC, août 2010.

12 Convention pour la répression d'actes illicites dirigés contre la sécurité de l'aviation civile Montréal, 23 septembre 1971; Protocole pour la répression d'actes illicites de violence dans les aéroports servant à l'aviation civile internationale, complémentaire à la Convention pour la répression d'actes illicites dirigés contre la sécurité de l'aviation civile, Montréal, 24 février 1988; Convention de l'OUA sur la prévention et la lutte contre le terrorisme, Alger, 14 juillet 1999.

13 CONFERENCE INTERNATIONALE SUR LA REGION DES GRANDS LACS, Paix et Sécurité, Projet sur la criminalité transnationale et le terrorisme, Fusion des Projets No. 1.3.1 et 1.3.2, Révision du 30 juin 2005. 
occuper ces installations ou ses biens ou à s'en emparer, ou à mettre en danger une ressource nationale $»^{14}$.

Cette étude s'intéresse à cette seconde forme de terrorisme en Afrique centrale et principalement en RDC, qui en réalité s'attaque à l'existence même de l'Etat contemporain dans lequel la notion de «l'Etat de droit» est devenue une référence incontournable de l'organisation politique ${ }^{15}$. Le Danger, et qui toute même est la raison fondamentale qui impose de réfléchir en profondeur sur cette question, est que ce fléau est un cycle infernal : de causes (Faillite de l'Etat et mauvaise gouvernance) aux conséquences (Effondrement de l'Etat - le chaos) et vice-versa qui menace l'organisation et le fonctionnement effectives de l'Etat. Il s'avère que la RDC se retrouve aujourd'hui dans un environnement régional pollué par l'islam radical (I) et pourtant, prenant en compte les exigences de cette « menace ${ }^{16}$ », une résilience efficace (II) de la RDC, n'est pas au rendez-vous. Dans cet état des choses, la menace est grave et imminente pour l' " Etat ${ }^{17}$ » en RDC d'autant plus que de vastes zones du pays connaissent aujourd'hui une absence du pouvoir régalien de l'Etat en dépit des efforts nationaux pour le développement et la consolidation de la présence de l'Etat et de l'Etat de droit en RDC.

\section{A. L'islam radical et violant en Afrique centrale : vecteur du terrorisme en RDC}

L'extrémisme islamiste violant s'est imposé parmi les préoccupations sécuritaires en Afrique centrale à cause d'une interconnexion et une exportation croissante et inévitable des activités criminelles caractérisant les terroristes islamistes radicaux. Le groupe Boko Haram, et finalement groupe Etat islamique en Afrique de l'ouest depuis le 7 mars 2015 ${ }^{18}$, s'impose comme l'un des principales menaces terroristes en Afrique qui part du Nigeria jusqu'au bassin du lac Tchad et au Cameroun, au Tchad et au Niger. Et en plus, ces terroristes du groupe Boko Haram ne s'empêchent pas d'atteindre la Centrafrique vers le Parc national de Bamingui Bangoran par le biais des alliances avec les rebelles de Seleka. Il faut également remarquer que le groupe terroriste somalien « Harakat al-Shabaab al-Mujahi-

14 Art. $1^{\text {er }}$ (2), Convention arabe relative à la répression du terrorisme, Caire, le 22 avril 1998. En vigueur depuis le 7 mai 1999.

15 RIVERO J., «L'Etat moderne peut-il être encore un Etat de droit? », Cairn, 2003, pp. 65-101.

16 Ici, il s'agit du terrorisme, menace à laquelle il n'est pas rationnel de déclarer la guerre. Car cette guerre serra perdue à l'avance. Une intervention militaire doit être circonscrite, avec un objectif ciblé, déterminée et limité pour qu'elle puisse être efficace et que toute nation peut utiliser avec raison et de la façon la plus métrisée possible. Mais il faut le dire, le terrorisme est une main invisible, mutante, opportuniste et on ne se bat pas contre une main invisible et asymétrique avec les armes de la guerre. Il faut être en mesure d'employer la force de l'esprit, de définir rationnellement des stratégies de la paix pour désolidariser ces forces qui s'agglutinent autour du noyau des terroristes.

17 NTUMBA-LUABA LUMU, Droit constitutionnel général, Kinshasa, 2007, p.30.

$18 A F P$, L'État islamique accepte l'allégeance de Boko Haram, Le monde, disponible sur http://www. huffingtonpost.fr/, consulté le 21 aout 2015. 
deen », qui sévit l'Afrique de l'Est ne ménage aucun effort pour attaquer au-delà de la Somalie jusqu'à atteindre l'Afrique centrale avec l'aide de l'ADF/NALU ougandais majoritairement constituée des musulmans ${ }^{19}$. Il sera question à ce niveau de présenter l'état des lieux de la situation (A), les causes et les conséquences de cette menace (B) dans la région.

\section{Etat des lieux : une Région havre et vivier du terrorisme}

L'Afrique centrale est prise entre les griffes du terrorisme qui parait aujourd'hui comme une nouvelle menace contre la paix et la sécurité dans cette région et surtout avec l'avènement du 11 septembre 2001, qui selon certains constitue la véritable rupture entre le $20^{\text {ème }}$ siècle et le $21^{\text {ème }}$ sicle, date à laquelle rien ne pouvait plus rester comme avant : les priorités des Etats comme celles de la Communauté internationale ont été bousculées, bafouées ou réformées ${ }^{20}$ suite aux drames macabres du terrorisme.

Le danger immédiat est véhiculé par une sorte de révolution sociale en provenant de l'Ouest de l'Afrique principalement du delta du Nigeria ${ }^{21}$, avec le groupe terroriste de Boko haram, aujourd'hui Groupe Etat islamique en Afrique de l'Ouest, qui atteint directement ou indirectement l'Afrique centrale. Cela s'aperçoit à travers l'existence et la survit des apparitions et des connexions avec les criminels Djinjahouids et des rebelles musulmans de la Seleka respectivement entre les frontières soudanaises, tchadienne et Centrafricaines notamment et avec lesquels il y a une économie de guerre qui est mise en place dans cette dynamique. En outre, aux côtés d'une rébellion de la LRA qui se réclame être de tendance chrétienne, on trouve une autre à tendance musulmane de l'ADF-NALU tous d'origine ougandaise qui ne s'empêchent de conclure des alliances avec les Al-Shabaab somaliens pour qui ces derniers combattent dans leurs rangs ${ }^{22}$. Telle est la cartographie des menaces qui portent main-forte aux terroristes auxquels l'Afrique centrale est confronté.

\section{Boko haram : un mutin en Afrique et aux portes de la RDC}

Boko Haram, qui signifie « l'éducation occidentale est un pêché », a été fondé vers 2002 par Mohamed Yusuf, activiste autodidacte, qui avait été instruit par la Muslim Students Society of Nigeria (Société des étudiants musulmans du Nigéria), MSSN en sigle, fondée en

19 BRAECKMAN C., « Kivu: des recrutements qui posent question », Le carnet de Colette Braeckman, mars 2015; RIGAUD C., RDC: des djihadistes au Kivu?, Afrikarabia, mars 2015, pp. 3-4.

20 NYABIRUNGU mwene SONGA, « Le blanchiment de capitaux et le financement du terrorisme. Exposé au Séminaire de fin-Consult du 29 au 30 avril 2009 », Annales de la Faculté de Droit de l’Université de Kinshasa, Kinshasa, Juin 2014, pp. 404-447.

21 La motivation de la naissance de Boko haram a été accéléré par l'injustice dans la répartition de la rente pétrolière entre les régions du nord et du sud du Nigeria, cf. ZENN J., « Leadership Analysis of Boko Haram and Ansaru in Nigeria », CTC Sentinel, vol.7, $n^{\circ} 2$, février 2014, p.23, disponible sur le cite https://www.ctc.usma.edu/wp-content/uploads/2014/02/CTCSentinel-Vol7Iss2.pdf., consulté le 20 mai 2014.

22 BRAECKMAN C., 19, p.13. 
1954, et plus particulièrement par Sheik Ibrahim Zakzaki ainsi que l'érudit salafiste Sheik Jafar Mahmoud Adam. Mohamed Yusuf s'est par la suite engagé dans sa propre voie idéologique, se démarquant des courants islamistes et salafistes dominants au Nigéria. Avec le même objectif que les djihadistes précédents, à savoir l'islamisation totale du Nigeria, telles sont les racines lointaines de Boko Haram, qui affirme que la corruption généralisée au sein du gouvernement, l'exclusion de la vie politique et les inégalités sociales disparaîtraient si le pays adoptait son concept rigoriste de l'Islam ${ }^{23}$. Cette précision vaut toute son importance aujourd'hui dans la lecture de son allégeance à l'EI qui n'est pas une surprise mais plutôt une matérialisation ou une manifestation de son caractère mutant et hybride de part l'origine de son idéologie initiale. Il est constaté que Boko haram est, en 2014, l'organisation la plus sanguinaire et redoutable du monde ${ }^{24}$. Il a fait allégeance à Al-Qaïda et à sa branche magrébine (AQMI), au Mouvement des talibans et finalement à l'EI, pour tout, Boko haram fait allégeance au plus offrant d'opportunités.

Boko Haram était au départ une secte religieuse, dont la radicalisation progressive a abouti à l'émergence d'un groupe extrémiste violent, se réclamant de la tradition talibane de type afghan, et ayant versé dans le terrorisme de masse. Boko Haram promeut un agenda les plus obscurs, qui mêle dans un indissociable cafouillis, l'imposition de la Sharia sur l'ensemble du Nigeria pour l'installation d'un Kalifa en Afrique de l'Ouest, à des revendications économiques et sociales de tout ordre fondées sur la répartition des revues pétrolières dans la région.

L'on peut décrire Boko Haram comme une secte dont les croyances se distancent de celles de la majorité des musulmans nigérians non radicaux. Dénonciatrice de la civilisation occidentale, rejette également la légitimité de l'État laïque nigérian, constamment décrit comme dagut (le « mal »), indigne de loyauté c'est pourquoi il s'est engagée en guerre contre ce gouvernement, pour tenter de lui substituer un régime islamique «purifié » basé sur une stricte observance de la Charia ${ }^{25}$. Mohamed Yusuf, le fondateur de Boko Haram et premier leader du groupe, était tué le 30 juillet 2009, suite à une grande opération militaire menée par l'armée nigériane, au court de laquelle près de 800 membres du groupe ont été également éliminés ${ }^{26}$. Depuis 2010, le chef du groupe est Abubakar Shekau. Il était auparavant le second de Mohamed Yusuf.

23 ZENN J., "Leadership Analysis of Boko Haram and Ansaru in Nigeria », CTC Sentinel, février 2014, vol.7, n², pp. 17-23, disponible sur le site https://www.ctc.usma.edu/wp-content/uploads/20 14/02/CTCSentinel-Vol7Iss2.pdf., consulté le 20 avril 2014.

24 GUIBBAUD Pauline, BOKO HARAM Histoire d'un islamisme sahélien, Paris, L'Harmattan, 2015, pp.7-13; Global Terrorism Databased: GTD Variables \& Inclusion Criteria (Base de données du terrorisme mondial : variables et critères d'inclusion dans la GDT). National Consortium for the Study of Terrorism and Responses to Terrorism (START), mai 2015; http://www.start.umd.edu/gtd /downloads/Codebook.pdf, consulté le 26 novembre 2015. 
Boko Haram est a priori focalisé sur un agenda national en dépit de sa tendance régionale ${ }^{27}$. Il semble toutefois s'être rapproché, au cours des dernières années, des groupes jihadistes sévissant sur l'espace sahélo-saharien ${ }^{28}$. De nombreux liens ont été tissés avec AlQaïda au Maghreb islamique (AQMI) et avec le groupe Al-Mourabitoune (MUJAO), dirigé probablement, au moins en partie, par Mokhtar Belmokhtar ${ }^{29}$. Boko Haram et d'autres groupes islamistes violents ont réussi à exploiter les griefs au sujet de la pauvreté généralisée, de la corruption au sein du gouvernement, des divisions ethno-religieuses et des abus commis par les forces de sécurité pour alimenter l'essor de la radicalisation islamique dans le nord du Nigeria. Le groupe acquiert une visibilité internationale accrue par l'enlèvement d'environ 276 lycéennes à Chibok au sud de l'État de Borno le 14 avril 2014.

En plus, le lien entretenu par Boko haram et d'autres groupes terroristes en Afrique est incontestable puisqu'en réalité, de nombreuses déclarations et arrestations de membres de Boko Haram et d'AQMI confirment des liens opérationnels, dont l'entraînement de djihadistes de Boko Haram par les katibas (brigades) d'AQMI ${ }^{30}$. Néanmoins, l'exagération autorisée par Abubakar Shekau, s'en prenant également aux populations civiles voire même au Nigeria, a conduit à quelques dissidences et à la constitution du groupe concurrent Ansar Al-Charia "Ansaru » en janvier 2012 ${ }^{31}$. Il existe une divergence de taille entre les deux groupes qui est la cible des attaques. Ansaru, déjà dans son agenda prend en compte un territoire plus vaste que le seul Nigeria, car ce groupe entend «défendre les musulmans africains» en général au contraire de Boko Haram qui focalise ces attaques sur le Nigeria principalement malgré l'exportation de ses attaques vers les Etats qui viennent en aide au Nigeria. Si des tensions ont été observées entre les deux groupes, ils semblent à présent coopérer, du moins constamment, et les résultats de leurs coopérations s'étendent au-delà des frontières nigérianes ${ }^{32}$.

27 BERGHEZAN G., 26, p.9.

28 PEROUSE DE MONTCLOS Marc-Antoine, Nigeria's Interminable Insurgency? Addressing the Boko Haram Crisis, Chatham House, Africa Programme, septembre,2014, pp. 3-7.

29 LEBOVICH Andrew, « Of Mergers, MUJAO, and Mokhtar Belmokhtar», al-Wasat, 23 août 2013, http://thewasat.wordpress.com/2013/08/23/of-mergers-mujao-and-mokhtar-belmokhtar/.

30 ADAM NOSSITER, «In Nigeria: A Deadly Group's Rage Has Local Roots », New York Times, 26 février 2012; lire également SERGE D., AQMI. Al-Qaïda au Maghreb islamique. L'industrie de l'enlèvement, Paris, 2012, pp.204-207.

31 ZENN J., "Leadership Analysis of Boko Haram and Ansaru in Nigeria », CTC Sentinel, février 2014, vol.7, n², p.23, disponible sur le cite https://www.ctc.usma.edu/wp-content/uploads/2014/0 2/CTCSentinel-Vol7Iss2.pdf., consulté le 20 mai 2014.

$32 \mathrm{PSC} / \mathrm{AHG} / 2 .(\mathrm{CDLV})$, Rapport de la président de la commission de 1'Union africaine sur le terrorisme et l'extrémisme violent en Afrique, 455ème réunion, au niveau des Chefs d'Etat et de gouvernement, Nairobi, 2 septembre 2014, p.2. 


\section{Al-Shabaab : une menace à multiple colorations}

Le groupe terroriste le plus évolué et le plus actif à l'Est de l'Afrique est le groupe terroriste somalien « Harakat al-Shabaab al-Mujahideen », qui parvient à conduire ses activités criminelles allant de la Corne de l'Afrique jusqu'au Sud de l'Afrique vers le Canal de Mozambique. Al-Shabaab qui signifierait « jeunesse » est un groupe terroriste islamiste somalien d'idéologie salafiste créé en 2006 lors de l'invasion éthiopienne. Le groupe est issu de l'Union des tribunaux islamiques et s'est fixé pour mission l'instauration de la charia dans la région. L'organisation est placée sur la liste officielle des organisations terroristes de l'ONU ${ }^{33}$.

Ce groupe terroriste entretient des liens forts avec la direction centrale d'Al-Qaïda au Pakistan et abrite des djihadistes étrangers, dont certains en provenance d'Europe et des États-Unis. L'intensification des actions militaires américano-pakistanaises dans les zones tribales au sud du Waziristân, une région montagneuse située au nord-ouest du Pakistan, a notamment entraîné le départ de plusieurs chefs d'Al-Qaïda vers la Somalie ${ }^{34}$.

Il y a plusieurs façons de considérer les islamistes shebaab. On peut dire que c'est un groupe affilié à Al-Qaïda. Une vision qui n'est pas fausse, mais c'est une vision étriquée de la réalité. Il faut considérer ce groupe comme l'extrême d'un spectre d'une organisation de l'islam politique somalien ${ }^{35}$. C'est un groupe qui s'est construit contre d'autres organisations armées salafiste dans un rapport de compétition mais aussi parfois d'alliances, d'opportunités et de rivalités en Somalie. Les types de recrutement ont été différents selon les périodes : parfois l'idéal Al-Qaïda dominait, parfois c'était la volonté de défendre la nation somalienne. A d'autres moments encore, les personnes intégraient l'organisation à cause de conflits locaux, ils voulaient profiter de l'organisation pour gagner localement un meilleur rapport de force ${ }^{36}$.

On se rend compte qu'il y a une complexité des points de vue qui est très bien reflétée par le mode de gouvernance des zones qu'ils contrôlent. Il y a de vraies différences sur la façon dont ils appliquent la charia et comment ils se comportent avec les populations. Il faut se rappeler que ce n'est pas simplement un groupe terroriste, ce sont aussi des gens qui gouvernent, qui prélèvent des taxes, qui distribuent, qui créent des services. On peut les comparer avec les MUJAO qui sont restés quelques mois dans la région de Gao au Mali.

33 UN, The List established and maintained by the Al-Qaida Sanctions Committee with respect to individuals, groups, undertakings and other entities associated with Al-Qaida, Last updated on 26 August 2014, disponible sur le cite http://www.un.org/sc/committees/1267/delisting.shtml., consulté le 02 septembre 2014.

34 SADIA SULAIMAN, " Pakistan: les nouveaux talibans entre deux feux », Alternatives Internationales $\mathrm{n}^{\circ}$ 041, décembre 2008, pp. 4-7.

35 HASSAN DJAMA FARAH, « Djibouti : un hub anti-piraterie et un acteur principal de la médiation dans la crise somalienne ", Diplomatie, un autre regard sur le monde, n56 - Mai-juin 2012, pp. $3-5$.

36 OGOULAT A-D., « Afrique Centrale et Golfe de Guinée : Géopolitique des Termes de l'Echange entre deux Regionymes Sous-contentieux », Enjeux, n² 26, 2006, p.13. 
Mais malgré les petites et les grandes internes, ils sont unis. De façon globale, ils partagent une vision de l'islam très salafiste, très rigoriste. Mais encore une fois, compte tenu des raisons très diverses qui poussent les gens à devenir Shebaab, les objectifs peuvent évoluer aussi ${ }^{37}$.

A l'absence de l'Etat en Somalie, s'ajoute l'exportation de la menace terroriste en représailles aux Etats de la région, ennemis de la Somalie selon l'idéologie du groupe AlShabaab. Les potentiels destinataires de l'exportation des activités terroristes d'Al-Shabaab en Afrique de l'Est sont principalement les pays de la région qui contribuent aux activités des Missions internationales de maintien de la paix en Somalie et les territoires voisins où l'autorité de l'Etat est quasi inexistante. Ici, le Kenya, la RDC, la Tanzanie et l'Ethiopie sont les plus grandes cibles : les uns participent aux missions internationales de maintien de la paix et les autres ont des vastes territoires sans pouvoir régalien de l'Etat. Il ne faut pas perdre de vue aussi que les Al-Shabaab ont fait allégeance à l'État islamique, comme l'ont fait les islamistes de Boko Haram ${ }^{38}$ qui sèment la terreur du Nigeria jusqu'en Afrique centrale à telle enseigne que la connexité opérationnelle entre ces groupes parait évidente. Un véritable danger aux portes de la RDC.

Dans le cycle de violence orchestré par des groupes rebelles armés dans la région des Grands Lacs, une grandissante menace islamiste est vécu dans la partie Est de la RDC au regard de la jonction qui se constate entre les groupes armées tentés par la propension des mouvements terroristes islamistes des ADF/NALU, des FDLR, de la LRA et même les récidivistes rwandais du M23 aux rangs desquels les Shebaab ne s'empêchent de combattre ${ }^{39}$. Quant à la recrudescence de violences dans le Nord-est de la RDC, certaines autorités politiques, affirment que de rebelles islamistes Shebaab sont entrés en action aux côtés des groupes armés qui pullulent dans l'Est du pays, notamment le M23 et l'ADF-NALU ${ }^{40}$ qui à leurs passage, sèment la terreur dans les régions constituant leur champ de bataille.

\section{Causes et conséquence de la recrudescence du terrorisme dans la région}

Les causes profondes de la recrudescence de la criminalité et la radicalisation violente de l'islam en Afrique, comme partout dans la plupart de régions, ne sont pas à recherche loin de l'organisation et du fonctionnement de l'Etat contemporain. Il suffit d'analyser en pro-

37 DIFFALAH Sarah, "Les shebab ne sont pas qu'un groupe terroriste, Politique africaine, disponible sur http://tempsreel.nouvelobs.com/, consulté le 21 aout 2015.

38 Jeune afrique, « Nigeria : Boko Haram fait allégeance au groupe État islamique », 8 mars 2015, disponible sur http://www.jeuneafrique.com., consulté le 21 aout 2015.

39 LUMUMBA R., RDC / Shebaab ou Boko Haram: Une nouvelles base arrière des islamistes à l'Est du Congo avec des recrutements tout azimut dans les mosquées et lieu de prière Musulman, disponible sur http://www.alterinfo.net, consulté le 21 aout 2015.

40 KIBANGULA T., RDC - Julien Paluku : «Les islamistes Shebaab combattent aux côtés du M23 et de l'ADF-Nalu », Jeuneafrique, 16 juillet 2013, disponible sur http://www.jeuneafrique.com, consulté le 5 février 2014. 
fondeur l'origine ou la naissance des groupes criminels ${ }^{41}$ pour s'en rendre compte. Les éléments qui reviennent pour la plupart sont notamment : la réaction contre l'injustice sociale, protestations contre la mauvais répartition des revenues nationales notamment la manne pétrolières dans les Etats pétroliers, la dictature accrue et notoire des dirigeants détenteur du pouvoir politiques, la pauvreté persistante et le désespoir d'une jeunesse désœuvrée qui manque à quel sort se vouer, la barbarie de politiques ${ }^{42}$. C'est la faillite de $1^{\prime} E^{2} t^{43}$, de la Gouvernance $^{44}$ : la mauvaise gouvernance. Et cette dernière favorise les trafics illicites de drogues, de substances psychotropes, de minerais, d'ivoires et d'espèces animales rares, voire même le trafic d'êtres humains, le blanchiment d'argent, la prolifération des armes légères et de petit calibre, tous ceux-ci constituant une autre série des causes qui, avec le terrorisme, constituent un cycle infernale.

En Afrique centrale, ce fléau est suspendu au dessus des Etats comme l'épée de Damoclès qui ne laisse émerger aucun effort ou initiative de développement de l'Etat à telle enseigne que ces pays dont les richesses naturelles minières, hydriques, forestières et les terres arables peuvent nourrir et développer l'Afrique tout entière, soit incapable de fournir le minimum à la grande majorité de ces habitants et d'éradiquer le sous-développement à l'intérieur de leurs propres frontières et de devenir le pilier et ressort pour le développement du Continent ${ }^{45}$.

La République Démocratique du Congo occupe une position géostratégique exceptionnelle au cœur de l'Afrique. Volontairement ou involontairement, elle est impliquée dans la quasi-totalité d'activités qui se déroulent chez ses voisins ou d'initiatives de ces derniers. Tout en ayant à l'esprit que les pays voisins de la RDC sont au nombre de neuf, essentiellement de l'Afrique centrale et enferment la RDC avec le seul trou d'aération étant cependant l'embouchure du fleuve Congo, il reste vrai que de la stabilité de RDC dépend celle de ses neufs pays voisins et vice-versa. Non sans raisons, l'écrivain Frantz Fanon avait présenté ce pays comme la « gâchette » de ce continent africain en forme de revolver.

L'Afrique centrale, avec environ une douzaine d'Etats, la RDC à elle seule compte neuf voisins. Cependant, une des particularités de l'instabilité de cette région, il faut le dire, est la coexistence de groupes criminels extrémistes islamistes et de groupes criminels ne se réclamant pas de l'islam extrémiste radical et violant. Ainsi, il y a une permanente menace

41 JIHAD GILLON et MARBOT O., «Tout sur "l'Etat islamique". Son histoire-Ses chefs-Ses ressources-Ses objectifs », La Revue pour l'intelligence du monde, Bimestriel, n`55-56, septembreoctobre 2015, pp. 18-37.

42 JIHAD GILLON et MARBOT O., 41, p. 21.

43 DIDIMALA LODGE, « Les Etats fragiles en Afrique: un paradigme utile pour l'action?, Rapport de conférence », Observatoire de 1'Afrique, Aout 2008, pp. 3-5.

44 NZONGOLA-NTALAJA G., Faillite de la gouvernance et crise de la construction nationale au Congo-Kinshasa. Une analyse des luttes pour la démocratie et la souveraineté nationale, KinshasaMontréal-Washington, ICREDES, 2015; GUINCHARD S. (dir.), Lexique des termes juridiques, $19^{\text {ème }}$ édition, Paris, 2012, p. 428.

NZONGOLA-NTALAJA G., 44, p.28. 
islamiste dans les régions Est de la RDC et dans la forêt équatorial à l'extrême Nord au regard de la jonction et connexion qui se constatent entre les groupes criminels armées tentés par le gout des alliances des mouvements terroristes islamistes avec les chefs des ADF/ NALU, les FDLR, la LRA. La RDC, qu'elle le veuille ou non, l'existence des uns et des autres dans la région met à mal la paix, la sécurité et la stabilité sur son territoire avec comme conséquence logique, des violations massives des droits de l'homme qui ne favorisent pas l'émergence de l'Etat de droit sur le territoire national.

Les enjeux majeurs du terrorisme sont essentiellement politiques et économiques. Il est vrai que les méthodes, les modes opératoires, les cibles et les victimes des terrorismes islamistes radicaux laissent certaines opinions dans une confusion inouïe. Mais, il faut le dire, c'est le pouvoir politique et le contrôle des ressources économiques qui sont visés, c'est l'objectif final. La RDC, qualifiée de scandale géologique en ce qui concerne les ressources minières, et l'instabilité politique qui y règne ${ }^{46}$, entretiennent un environnement favorable aux criminels, il devient sans aucun doute l'épicentre d'une région propice au développement du terrorisme.

Il faut également y ajouter un certain nombre de facteurs de vulnérabilité qui ont élu domicile en RDC et constituent des circonstances aggravantes de la situation. Du nombre de ces facteurs, il y a l'immensité du territoire national, sa géographie physique, avec des vastes régions hors de l'autorité de l'Etat qui constituent les sanctuaires de la criminalité. L'absence de l'exercice du pouvoir régalien de l'Etat dans une région engendre inévitablement un environnement favorable au terrorisme. Les forêts de la RDC, couvrant une superficie estimée à 155,5 millions d'ha (dont 99 millions d'ha de forets denses), soit $67 \%$ du territoire national rattrapées par cette réalité n'échappent pas à l'expansion des islamistes radicaux exportateurs du terrorisme ${ }^{47}$.

Une porosité des frontières donne lieu à d'incessants mouvements transfrontaliers difficiles à contrôler par les administrations d'États de l'Afrique centrale et l'insécurité croissante aux frontières nationales sont autant des facteurs qui rendent la RDC et toute la région menacées plus que jamais du terrorisme et de manière générale, de la criminalité transnationale organisée ${ }^{48}$ d'autant plus que les nébuleuses trouvent des vastes zones à l'abris de toute inquiétude et perturbation de leurs activités criminels. C'est ainsi que les vastes forets de la région équatoriale au nord de la RDC, en Centrafrique jusqu'au Soudan du Sud servent de base-arrière pour les ADF-NALU, la LRA, la FDLR aux rangs desquels les She-

46 NZONGOLA NTALAJA G., 44, pp. 225-361.

47 EBA’A ATYI R. et BAYOL N., «Les forets de la République démocratique du Congo en 2008 », observatoire-comifac, $\mathrm{n}^{\circ} 07$, juillet 2008, pp. 115-128.

48 NDESHYO RURIHOSE O. et ABDOU MUHIMA M., La criminalité transnationale organisée en Afrique : une grave menace contre la paix et la sécurité des africains, Revue internationale de criminologie et de police technique et scientifique, Vol. LXVIII, Genève, Avril-Juin 2015, pp. $131-157$. 
baab somaliens ne s'empêchent de combattre ${ }^{49}$ et l'Etat islamique en Afrique de l'Ouest ne ménage aucun effort pour atteindre l'Afrique centrale vers les vastes régions forestières de la République centrafricaine ${ }^{50}$.

Le développement du terrorisme laisse transparaitre un cycle de violence entretenu principalement par les détenteurs du pouvoir politique et ceux qui ont été évincé du cercle de l'impérium. Les groupes criminels qui entretiennent et nourrissent le terrorisme en Afrique centrale sont pour la plupart des groupes rebelles armés qui multiplient des alliances avec les groupes terroristes de l'Est à l'Ouest. C'est ainsi que les terroristes de l'Etat islamique en Afrique de l'Ouest arrive jusqu'en Afrique centrale par le biais des alliances avec des groupes rebelles de la Seleka en Centrafrique. De même, les Shebaab somaliens arrivent jusqu'à l'Est de la République démocratique du Congo, où ils combattent aux côtés des groupes rebelles armés qui déstabilisent la région ${ }^{51}$.

Les activités de groupes terroristes en Afrique centrale font des populations d'objets que l'on peut à tout moment manipuler ou jeter au rebut au gré de ses humeurs au mépris des lois et des exigences humanitaires. Il y a en premier lieu le nombre élevé de réfugiés que la région connait en ce jour. En examinant les chiffres provenant de la part des organisations internationales humanitaires ${ }^{52}$, la situation est toujours déplorable d'autant plus que les activités des groupes terroristes dans la région, principalement l'Etat islamique en Afrique de l'ouest (ex-Boko haram) et le Shebaab, ont notamment poussé quelque 190000 personnes à fuir leurs résidences habituelles. Ces mouvements obligés des populations s'observent notamment au Cameroun, au Tchad, au Niger, en Centrafrique, voire même au Nigeria, bien que ce dernier n'est pas de l'Afrique centrale mais constitue un sanctuaire principal du terrorisme qui menace plus que jamais les pays de l'Afrique centrale ${ }^{53}$.

Cet état de la situation prouve à suffisance que l'Afrique centrale est malheureusement devenue aujourd'hui la région d'errance massive des populations. Ce mouvement intermittent et non voulu, fait des femmes, des hommes et des enfants, des personnes sans terre ni patrie, dépouillées de toute identité si ce n'est que celle d'avoir un numéro dans les camps des réfugiés ou des déplacés afin d'obtenir leur hypothétique pitance journalière à condition que la guerre ne les remettent pas chaque fois de nouveau en marche ou ne les fasse à ja-

49 LE POTENTIEL, « RDC : Les FARDC affrontent aussi les islamistes ougandais et Shebaab somaliens au Nord-Kivu », disponible sur http://www.lepotentielonline.com/.

50 BIDA KOYAGBELE P., Centrafrique : Boko haram et l'état islamique (EI) au cœur de l'Afrique, JNRD, 2015, pp. 8-9.

51 FIORINA J.-F., « Boko Haram : religion et frontières en Afrique. Secte islamiste, gang criminel ou rébellion ethnique? », Comprendre les Enjeux Stratégiques, n¹51, Février 2015, pp.1-3..

52 UNHCR, The World", UNHCR, 2011, EOUZAN J.P., Déplacements de populations et trypanosomiase humaine en Afrique centrale, International Journal of Tropical Insect Science, Vol.1, 19 September 2011.

53 C.S, Rapport du Secrétaire général sur la situation en Afrique centrale et les activités du Bureau régional des Nations Unies pour l'Afrique centrale, 7461e séance, le 11 juin 2015. 
mais disparaître ${ }^{54}$. Les effets d'un tel déplacement forcé, engendrent la famine, les paysans étant empêchés de l'exercice de leurs travaux champêtres pour subvenir autant à leurs propres besoins qu'à ceux de leurs concitoyens. Les populations ainsi déplacées errent dans une précarité inouïe autant sur le plan physique que moral au point de devenir des mortsvivants confrontés à des maladies de tous genres qui viennent remplir, le lugubre tableau de maux. Sur le plan social, une désintégration totale des familles se vit dans les camps de réfugiés et des déplacés. Le concept de famille perd tout son sens. Drame douloureux pour l'Afrique central que les protagonistes et les commanditaires de ces sales guerres se refusent à regarder en face et d'arrêter.

Autre conséquence tout aussi terrible et intolérable dans ces conflits est l'enrôlement d'enfants dans les rangs des bandes combattantes que pratique sans scrupule la LRA avec plus de $80 \%$ dans ces effectives continué des mineurs ${ }^{55}$ et d'autres groupes armés. Pourraiton alors penser un instant que des enfants puissent être sciemment drogués durant ces horribles conflits sans règles ni codes et jetés dans les rues au cours d'opérations prétendument militaires, tuant, volant et violant quel que soit leur âge? La guerre pour ces gamins en mal d'héroïsme est devenue un jeu mais un jeu macabre et le fusil tient lieu d'écritoire dont ils devraient normalement se servir à cet âge-là pour parfaire leurs connaissances à l'école.

Le fonctionnement des Etats de la région ne sont pas moins touchés par les activités terroristes et les groupes criminels qui sévissent en Afrique centrale. La recrudescence des activités terroristes dans la région constitue tout d'abord une preuve de l'ineffectivité de l'exercice du pouvoir régalien sur les territoires nationaux. Le blocage des activités étatiques par les activités terroristes ce manifeste dans toute la région. Le groupe Boko haram, aujourd'hui Etat islamique en Afrique de l'Ouest et le Al-Shabaab somalien avec leurs alliées constitués des groupes criminels ou rebelles armés sèment le chao organisationnel sur le chemin $^{56}$, sans raison qui pourrait être conforme aux exigences de la démocratie et de l'Etat de droit contemporain.

\section{B. La résilience de la RDC : une léthargie au péril de l'Etat}

La République démocratique du Congo ce doit de prendre des dispositions nécessaires pour renforcer ses capacités de prévenir et de réprimer le terrorisme. La nécessité de ce devoir se trouver encré dans l'ensemble des enjeux qui prévalent à la recrudescence du terrorisme dans le monde et particulièrement en Afrique. L'instabilité politique et le paradoxe de la précarité économique du pays, ne constituent que des signaux forts qui appellent à l'urgence d'une résilience efficace contre le terrorisme en RDC.

54 UNHCR, The World", UNHCR, 2011, pp. 14-19.

55 KAREN LAJON, « Joseph Kony, ennemi de l'humanité », JDD, 10 mai 2015; UNICEF, Les enfants soldats, Rapport 2012, Nicolas CLEMESAC, Comprendre le phénomène des enfants soldats, JRS, Bujumbura, février 2007.

56 SAURAL A., "Les élections au Nigeria perturbées par Boko Haram », Egalité et réconciliation, 28 mars 2015. 
Il est évident que la répression est un outil important pour la résilience contre ces fléaux, mais les règles, les mécanismes et les techniques de prévention et de détection s'avèrent nécessaire même si, en fin de compte, leur violation appelle des sanctions pénales ${ }^{57}$. C'est ainsi que dans cette dynamique de résilience s'inscrivent des initiatives pour la prévention contre ce fléau (A), aux côtés desquelles il est prévu des mesures répressives (B).

\section{Les initiatives de la République démocratique du Congo pour la prévention du terrorisme}

Puisqu'il est irréaliste de déclarer la guerre contre le terrorisme, cette main invisible, la prévention contre ce fléau parait rationnelle et réaliste pour une prise en charge efficace. Compte tenu des enjeux majeurs qui sous-tendent la recrudescence du terrorisme en Afrique, il est évident que la refondation de l'Etat dans toutes ses dimensions reste le remède idéal pour prévenir le terrorisme en Afrique. Cet impératif s'impose à la République démocratique du Congo, qui aux yeux des observateurs avisés, est loin d'être un Etat stable, qui exerce son autorité sur l'ensemble du territoire national ${ }^{58}$.

La prévention du terrorisme passe à travers des initiatives étatiques plurisectorielles. Le développement économique et socio-culturel, militaire, la bonne gouvernance, l'amélioration des conditions sécuritaires, voire même juridique sont notamment les secteurs sur lesquels l'Etat peut s'appuie pour prévenir la radicalisation des masses, et ainsi détruire l'économie de guerre sur laquelle le terrorisme se fonde pour mobiliser les recrutements, le financement et les bases-arrières de protection ${ }^{59}$. En République démocratique du Congo, certaines initiatives s'inscrivent dans cette optique, mais cette étude s'intéresse à la loi n ${ }^{\circ} 04 / 016$ du 19 juillet 2004 portant lutte contre le blanchiment des capitaux et le financement du terrorisme (1) et des institutions spécialisées mises en place par le Gouvernement pour la lutte contre ce fléau (2).

1. La loi $\mathrm{n}^{\circ} 04 / 016$ du 19 juillet 2004 portant lutte contre le blanchiment des capitaux et le financement du terrorisme en RDC

Pour prévenir le terrorisme, cette loi définie et sanctionne le financement de cette activité odieuse. Il se dégage que cette loi s'articule autour de quelques dispositions générales relatives à la prévention et à la détection d'actes constitutifs de financement du terrorisme. Il

57 NYABIRUNGU mwene SONGA, 20, p. 406.

58 BIYOYA MAKUTU KAHANDJA P., La géopolitique de l'instabilité dans la région des Grands lacs. Réflexion sur les réfugiés, ces acteurs/auteurs des mutations géostratégiques, Kinshasa, 2009, p.11 et ss.

59 GAULME F., «Les économies de guerre, nouveau mal du siècle », Études 2005/11 (Tome 403), p. 441-452. 
convient de noter qu'elle n'aborde que les questions spécifiques liées au financement du terrorisme et ne s'étend donc pas au phénomène « terrorisme» dans toute sa complexité60.

Cette loi définit le terrorisme comme étant, l'ensemble d'actes en relation avec une entreprise individuelle ou collective ayant pour but de troubler gravement l'ordre public par l'intimidation ou la terreur, à savoir :

a. les atteintes volontaires à la vie ou à l'intégrité physique de la personne, l'enlèvement et la séquestration de la personne ainsi que le détournement d'aéronefs, de navires ou de tout autre moyen de transport;

b. les vols, extorsions, destructions, dégradations et détériorations;

c. la fabrication, la détention, le stockage, l'acquisition et la cession des machines, engins meurtriers, explosifs ou autres armes biologiques, toxiques ou de guerre;

d. tout autre acte des mêmes natures et but consistant à l'introduction dans l'atmosphère, sur le sol, dans le sous-sol ou dans les eaux de la République, d'une substance de nature à mettre en péril la santé de l'homme ou des animaux ou le milieu naturel ${ }^{61}$.

Le législateur de la RDC s'appuie sur cette définition pour donner un sens au financement du terrorisme qu'il incrimine et définit comme étant le fait d'une part, de fournir, de collecter, de réunir ou de gérer par quelque moyen que ce soit, directement ou indirectement, des fonds, des valeurs ou des biens dans l'intention de les voir utilisés ou en sachant qu'ils seront utilisés, en tout ou en partie, en vue de commettre un acte de terrorisme indépendamment de la survenance d'un tel acte ${ }^{62}$.

Cette loi se préoccupe principalement de toute personne physique ou morale qui, dans le cadre de sa profession, réalise, contrôle ou conseille des opérations entraînant des dépôts, des échanges, des placements, des conversions ou tous autres mouvements de capitaux, et en particulier à la Banque Centrale du Congo; aux établissements de crédit, messageries financières, compagnies financières, institutions de micro-finance, bureaux de change, entreprises d'assurance, intermédiaires en assurance ou réassurance, entreprises de leasing et autres intermédiaires financiers; aux services comptes chèques et mandats postaux; aux bourses de valeurs mobilières, sociétés de bourses, intermédiaires en opérations de bourse, sociétés de gestion du patrimoine, entreprises offrant des services d'investissement et organismes de placement collectif en valeurs mobilières; aux sociétés de loteries ${ }^{63}$.

Elle s'applique en outre aux gérants, propriétaires et directeurs des casinos; aux notaires; aux membres des professions juridiques indépendantes, notamment les avocats, lorsqu'ils conseillent ou assistent des clients ou lorsqu'ils agissent en leurs noms et pour leurs comptes dans l'achat et/ou la vente de biens, d'entreprises ou de fonds de commerce, de titres ou d'autres actifs, la manipulation d'actifs, lors de l'ouverture des comptes bancaires,

60 Exposé des motifs de la loi nº4/016 du 19 juillet 2004 Portant lutte contre le blanchiment des capitaux et financement du terrorisme.

61 Art. 3 (8) de la loi nº4/016 du 19 juillet 2004, op.cit.

62 Art. 2 de la loi nº4/016 du 19 juillet 2004, Idem.

63 Art. 4 de la loi ${ }^{\circ} 04 / 016$ du 19 juillet 2004, Op.cit. 
la constitution, la gestion ou la direction des sociétés, des fiducies ou de structures similaires, ou de toutes autres opérations financières; aux agents immobiliers et autres conseillers en opérations immobilières; aux transporteurs de fonds; aux agences de voyage; aux commissaires aux comptes, experts comptables, auditeurs externes et conseillers fiscaux; aux marchands d'œuvres d'art, d'antiquités et/ou de matières précieuses ${ }^{64}$.

L'application de tout ou partie des dispositions de cette loi peut être étendue à toute autre profession ou catégorie d'entreprises lorsqu'il est constaté que cette profession ou catégorie d'entreprises réalise, contrôle ou conseille les mêmes types d'opérations précisées au paragraphe premier du présent article.

a) De la prévention et de la détection du blanchiment des capitaux

Pour la prévention du blanchiment de capitaux, la loi n04/016 du 19 juillet 2004 portant lutte contre le blanchiment des capitaux et le financement du terrorisme prévoie un seuil, en chiffre, maximum pour les opérations financières à effectuer en RDC. Elle exigence que tout paiement d'une somme en francs congolais ou autre globalement égale ou supérieure à 10000 dollars américains ne soit acquitté en espèces ou par titres au porteur. Il faut qu'une instruction du Gouverneur de la Banque Centrale du Congo détermine les cas et conditions auxquels une dérogation à cette restriction soit admise notamment pour les opérateurs économiques régulièrement inscrits au nouveau registre de commerce, pour les tenanciers des comptoirs d'achat des matières précieuses et leurs collaborateurs, pour les opérateurs agricoles et pour leurs employeurs ${ }^{65}$.

Cette loi fait une restriction mois suffisante aux transferts internationaux en exigeant que tout transfert vers l'étranger ou en provenance de l'étranger, de fonds, titres ou valeurs pour une somme égale ou supérieure à 10.000 dollars américains soit effectué par un établissement de crédit ou par son intermédiaire ${ }^{66}$, et pourtant, les établissement des crédit, voire même leurs intermédiaires, ne s'empêchent plus de financer ou de soutenir le terrorisme dans le monde de quelque manière que ce soit. Du nombre, est citée notamment la banque suisse HSBC Private Bank qui a cautionnée une gigantesque fraude Sà l'échelle internationale portant sur des milliards d'euros qui auraient transité, à Genève pour soutenir et financer des groupes terroristes ${ }^{67}$.

Pour s'assurer la transparence dans les transactions financières, le législateur exige aux établissements de crédits de mettre en place un dispositif de prévention du blanchiment de capitaux qui comprend la centralisation des informations sur l'identité des clients, donneurs d'ordre, bénéficiaires et titulaires de procuration, mandataires, ayants droit économiques, et

64 Idem.

65 Art. 5 de la loi n04/016 du 19 juillet 2004, Ibidem.

66 Art. 6 de la loi $n^{\circ} 04 / 016$ du 19 juillet 2004, Op.cit.

67 VEREYCKER Karel, "HSBC, voyage au cœur de la finance criminelle », Solidarité et progrès, mars 2015, pp. 1-9; Manuel Gomez, « La banque HSBC finançait le terrorisme », disponible sur www. http://magoturf.over-blog.com., consulté le 21 aout 2015. 
sur les transactions suspectes; la désignation des responsables de l'unité de centralisation auprès du siège ou de la direction centrale, de chaque succursale, et de chaque agence ou service local; la formation continue des fonctionnaires ou employés; et en fin, un dispositif de contrôle interne de l'exécution et de l'efficacité des mesures adoptées pour l'application de la loi portant lutte contre le blanchiment de capitaux et financement du terrorisme.

La mission de la détection du blanchiment de capitaux comme celui du financement du terrorisme en RDC est confiée au Ministère public, à la banque centrale du Congo, auxquels il faut ajouter les services spécialisés du gouvernement, principalement la Cellule Nationale des renseignements financiers (CENAREF) et le Comité consultatif de lutte contre le blanchiment de capitaux et le financement du terrorisme (COLUB) qui sont soutenus par le Fonds de lutte contre le crime organisé (FOLUCCO) ${ }^{68}$.

b) De la prévention et de la détection du financement du terrorisme

En République démocratique du Congo, toute personne physique ou morale qui, dans le cadre de sa profession, réalise, contrôle ou conseille des opérations entraînant des dépôts, des échanges, des placements, des conversions ou tous autres mouvements de capitaux a l'obligation de déclarer les opérations réalisées même s'il a été impossible de surseoir à leur exécution ou s'il n'est apparu que postérieurement à la réalisation de l'opération que celleci portait sur des fonds suspects de financement du terrorisme. Elles sont également tenues de déclarer, sans délai, toute information tendant à renforcer le soupçon ou à l'infirmer ${ }^{69}$.

Les déclarations de soupçons de financement du terrorisme sont adressées auprès de la Cellule des Renseignements Financiers et du Ministère public, lorsqu'elles suspectent que, d'une part, des fonds appartenant aux personnes ou entités reprises sur la liste des organisations considérées comme terroristes, celle des organisations à but caritatif, culturel ou social suspectées de tendance terroriste ainsi que celle des organisations impliquées notamment dans des activités de trafic illicite d'armes, de stupéfiants, de proxénétisme et de blanchiment de capitaux, établies conformément aux résolutions des Nations Unies relatives à la prévention et à la répression du financement des actes terroristes, ou, d'autre part, des mouvements de fonds initiés par elles ou pour leur compte, sont liés au financement du terrorisme ou destinés à être utilisés à cette fin ${ }^{70}$.

Toutes les personnes visées par la loi portant lutte contre le blanchiment des capitaux et financement du terrorisme pour dénoncer tout soupçon des fonds en provenance d'activités

68 Art. 31 de la loi nº4/016 du 19 juillet 2004, Op.cit.; Décret $n^{\circ} 08 / 20$ du 24 septembre 2008 portant organisation et fonctionnement d'une Cellule nationale des renseignements financiers " CENAREF »; Décret n $08 / 21$ du 24 septembre 2008 portant création du Comité consultatif de lutte contre le blanchiment de capitaux et le financement du terrorisme « COLUB »; Décret n ${ }^{\circ} 08 / 22 \mathrm{du}$ 24 septembre 2008 portant création du Fonds de lutte contre le crime organisé, en sigle « FOLUC$\mathrm{CO} »$.

69 Art. 20 de la loi n ${ }^{\circ} 04 / 016$ du 19 juillet 2004, Op.cit.

70 Art. 28, Idem. 
illicites ou pour financer ceux-ci, sont exemptées de toute responsabilité et aucune poursuite pour violation du secret professionnel ne peut être engagée contre eux ou leurs préposés qui, de bonne foi, ont transmis les informations ou effectué les déclarations visant à lutter contre le financement du terrorisme et le blanchiment de capitaux quelle que soit l'issue d'enquêtes ou de décisions judiciaires ${ }^{71}$. Mais aussi, les Etablissement des crédits ne peuvent en aucun cas invoquer l'obligation du secret professionnel pour refuser de fournir ou de procéder aux déclaration requises dans le cadre d'une enquête portant sur des faits de blanchiment ou de financement du terrorisme ordonnée ou effectuée par l'autorité judiciaire $^{72}$.

\section{Les institutions spécialisées pour la détection et la prévention du terrorisme}

Le gouvernement de la République démocratique du Congo a créée la Cellule nationale des renseignements financiers (CENAREF) et le Comité consultatif de lutte contre le blanchiment de capitaux et financement du terrorisme (COLUB) qui sont appuyées par le Fonds de lutte contre le crime organisé (FOLUCCO). Ce dernier, le FOLUCCO, a été créé en vue de la lutte contre le crime organisé notamment le trafic de drogue, le blanchiment des capitaux et le financement du terrorisme ${ }^{73}$.

Dans cette perspective, le FOLUCCO est destiné à financer l'organisation et le fonctionnement des structures chargés de lutte contre le crime organisé, notamment le terrorisme, et finance également la formation des agents des services publics et autres institutions de l'Etat impliqués dans la lutte contre ce type de criminalité et les études sur l'évolution des techniques utilises aux fins notamment de blanchiment des capitaux ou de financement du terrorisme sur le territoire national ${ }^{74}$. Les avoirs de ce fonds sont logés dans un compte ouvert à la Banque centrale du Congo et sont alimentés par les ressources et biens confisqués, dévolus à l'Etat, suivant les modalités fixées par un arrêté interministériels des Ministres ayant les Finances et la Justice dans leurs attributions ${ }^{75}$.

a) La cellule nationale de renseignements financiers (CENAREF)

La CENAREF est une institution spécialisée créée par le Gouvernement de la RDC pour appuyer les initiatives gouvernementales de lutte contre le blanchiment des capitaux et financement du terrorisme sur le territoire national.

71 Art. 24, Ibidem.

72 Art. 27, Ibidem.

73 Art. ${ }^{\text {er }}$ du Décret n ${ }^{\circ} 08 / 22$ du 24 septembre 2008 portant création du fonds de lutte contre le crime organisé, en sigle «FOLUCCO », Journal Officiel de la RDC, n²0, 15 octobre 2008.

74 Art. 2 du Décret n ${ }^{\circ} 08 / 22$ du 24 septembre 2008, Idem.

75 Art. 3 et 4 du Décret n ${ }^{\circ} 08 / 22$ du 24 septembre 2008, Ibidem. 


\section{i) Création et missions}

La CENAREF a été crée par la loi n04/016 du 19 juillet 2004 portant lutte contre le blanchiment de capitaux et financement du terrorisme. Elle est un service public à caractère administratif et technique dotée d'une autonomie financière, d'un pouvoir de décision propre et placée sous la tutelle du Ministre des Finances. La mission de la Cellule des Renseignements Financiers est de recueillir et de traiter les renseignements financiers sur les circuits de blanchiment de capitaux et de financement du terrorisme ${ }^{76}$. Elle reçoit aussi toutes autres informations utiles, notamment celles communiquées par les autorités judicaires. Elle fait poursuivre, le cas échéant, les personnes présumées coupables de blanchiment des capitaux et de financement du terrorisme ${ }^{77}$.

La cellule réalise ou fait réaliser des études périodiques sur l'évolution des techniques utilisées aux fins de blanchiment de capitaux et de financement du terrorisme sur le territoire national et émet des avis sur la politique de l'Etat en matière de lutte contre le blanchiment des capitaux et le financement du terrorisme, et sur sa mise en œuvre. A ce titre, elle propose les réformes appropriées au renforcement de l'efficacité de la lutte contre le blanchiment des capitaux et le financement du terrorisme ${ }^{78}$.

Elle élabore des rapports trimestriels sur ses activités. Ces rapports indiquent les techniques de blanchiment et de financement du terrorisme éventuellement relevées sur le territoire national et les propositions visant à renforcer la lutte contre le blanchiment de capitaux et le financement du terrorisme. Elle établit annuellement un rapport récapitulatif. Ces rapports dont copies sont réservées au Ministre de la Justice et au Gouverneur de la Banque Centrale du Congo, sont adressés au ministre des Finances ${ }^{79}$.

\section{ii) Organisation et fonctionnement}

Les organes de la CENAREF sont le Conseil et le secrétariat Exécutif, qui sont soutenus par un personnel dont le statut est fixé par le Conseil, sur proposition du Secrétariat Exécutif $^{80}$. Le conseil a les pouvoir les plus étendus pour poser des actes de disposition, d'administration et de contrôle. A ce titre, il adopte le budget et établit les comptes annuels, établi ou supprime des agences et bureaux. Le conseil définit le cadre organique et le statut des agents, et adopte les rapports trimestriels et annuels de la Cellule ${ }^{81}$.

Le conseil est composé de neuf membres jouissant d'une intégrité morale reconnue et ayant des compétences en matière de blanchiment des capitaux et financement du terro-

76 Art. 17 de la Loi nº4/016 du 19 juillet 2004, Op.cit; Art. 1 du Décret nº8/20 du 24 septembre 2008, op.cit.

77 Art. 3 du Décret n08/20 du 24 septembre 2008, Idem.

78 Art. 3 du Décret n ${ }^{\circ} 08 / 20$ du 24 septembre 2008, Op.cit.

79 Art. 17 de la Loi n ${ }^{\circ} 04 / 016$ du 19 juillet 2004, Op.cit.

80 Art. 6 et Art. 20 du Décret n08/20 du 24 septembre 2008, Idem.

81 Art. 7 du Décret $n^{\circ} 08 / 20$ du 24 septembre 2008, Ibidem. 
risme. Ils sont nommés par le Président de la République pour un mandat de six ans renouvelable, sur proposition du Ministre ayant les Finances dans ces attributions. Tous ses membres exercent leur mandat en temps partiel ${ }^{82}$.

Le secrétariat Exécutif est composé du Secrétaire exécutif et du Secrétaire exécutif adjoint. Ils dirigent et surveillent l'ensemble des services de la Cellule. Le Secrétariat exécutif veille à l'exécution des décisions du Conseil et assure la gestion courante de la CENA$\mathrm{REF}^{83}$. Dans le fonctionnement du la cellule, sauf disposition contraire expresse, le Ministère de tutelle, exerce son pouvoir par voie d'autorisation préalable ou d'approbation. Dans cette perspective, certains actes sont soumis au régime d'autorisation préalable et d'autres l'approbation ${ }^{84}$.

b) Le Comité consultatif de lutte contre le blanchiment de capitaux et le financement du terrorisme (COLUB)

Le Comité consultatif de lutte contre le blanchiment de capitaux et le financement du terrorisme a été crée pour assister le Gouvernement dans la définition et la mise en œuvre de la politique nationale de lutte contre le blanchiment des capitaux et le financement du terrorisme. Il propose au Gouvernement les mesures adéquates à prendre pour l'amélioration de la stratégie et du dispositif national de lutte contre le blanchiment des capitaux et le financement du terrorisme ${ }^{85}$.

Le Comité examine, à la demande du Gouvernement, les modalités et conditions de mise en œuvre en RDC des recommandations de la communauté internationale relative à la lutte contre le blanchiment des capitaux et le financement du terrorisme et assure une meilleure information des Services publics et professionnels impliqués dans cette lutte ${ }^{86}$. Le Comité consultatif est composé de vingt-huit membre dont seize au titre d'Institutions et services publics et douze au titre des professions assujettis à la loi $n^{\circ} 04 / 016$ du 19 juillet 2004 portant lutte contre le blanchiment des capitaux et financement du terrorisme ${ }^{87}$.

\section{La répression du terrorisme en République démocratique du Congo}

Dans la dynamique de la répression du terrorisme, le législateur congolais a distingué la répression du financement du terrorisme de celle de la répression du terrorisme en tant que tel. C'est ainsi qu'interviennent principalement deux textes de loi, le premier est la loi $n$

82 Art. 8, Ibidem.

83 Art. 16, Ibidem.

84 Art. 28, Ibidem.

85 Art. 2 du Décret $n^{\circ} 08 / 21$ du 24 septembre 2008 portant création du Comité consultatif de lutte contre le blanchiment des capitaux et financement du terrorisme, COLUB en sigle, Journal Officiel de la RDC, $n^{\circ} 20,15$ octobre 2008 .

86 Art. 2 Idem.

87 Art. 3 Idem. 
${ }^{\circ} 024 / 2002$ du 18 novembre 2002 portant code pénal militaire et le second est la loi $n$ ${ }^{\circ} 04 / 016$ du 19 juillet 2004 portant lutte contre le blanchiment de capitaux et financement du terrorisme. Le constat est que le terrorisme n'a pas fait objet d'incrimination dans le code pénal ordinaire en dépit des enjeux et des intérêts en présence ${ }^{88}$ sans justification, le législateur ordinaire.

\section{1) La répression du financement du terrorisme}

Le financement du terrorisme est alimenté par la masse d'argent tirée des circuits illégaux constituant des économies de guerre que les criminels tentent par tout les moyens à insérer dans le circuit légal des capitaux pour son usage. Le danger est que la République démocratique du Congo est considérée comme un lieu de refuge de l'argent sale ${ }^{89}$. De ce fait, les criminels s'emploient au blanchiment de capitaux pour financer entre autres le terrorisme.

En effet, la répression du financement du terrorisme est étroitement liée à la répression du blanchiment de capitaux, bien entendu que ces deux soient distinctes l'une de l'autre. Le blanchiment des capitaux étant lié au financement du terrorisme, seront punis de cinq à dix ans de servitude pénale et d'une amende dont le maximum est égal à six fois le montant de la somme blanchie, ceux qui auront commis un fait de blanchiment. A ce titre, le complice et le co-auteur de l'infraction du blanchiment sont punis des mêmes peines que l'auteur même de l'infraction de blanchiment ${ }^{90}$.

Le législateur congolais prévoit en outre que les personnes morales pour le compte ou au bénéfice desquelles une infraction de blanchiment de capitaux a été commise par l'un de leurs organes ou représentants, seront punies d'une amende d'un taux égal au quintuple des amendes spécifiées pour les personnes physiques, sans préjudice de la condamnation de ces dernières comme co-auteurs ou complices de l'infraction ${ }^{91}$. Et ces personnes morales peuvent également être condamnée à l'interdiction à titre définitif ou pour une durée de cinq ans au plus, d'exercer directement ou indirectement certaines activités professionnelles et à la fermeture définitive ou pour une durée de cinq ans au maximum, de leurs établissements ayant servi à commettre l'infraction. Elles peuvent en outre être condamnée à la dissolution lorsqu'elles ont été créées pour commettre les faits incriminés et au paiement des frais de publication de la décision par la presse écrite ou par tout autre moyen de communication audiovisuelle ${ }^{92}$.

En ce qui concerne le financement du terrorisme, le législateur prévoit qu'il sera punie d'une servitude pénale de cinq à dix ans et d'une amende en francs congolais équivalente à 50.000 dollars américains, toute personne physique auteur, co-auteur ou complice de l'in-

88 NYABIRUNGU mwene SONGA, 20, p.411.

89 Idem, p. 405.

90 Art. 34 de la Loi nº4/016 du 19 juillet 2004, Op.cit.

91 Art. 36 de la Loi n $04 / 016$ du 19 juillet 2004, Idem.

92 Art. 36 de la Loi nº4/016 du 19 juillet 2004, Ibidem. 
fraction de financement du terrorisme ${ }^{93}$. La commission de l'infraction dans le cadre d'une organisation criminelle en constitue une circonstance aggravante qui peut amener la peine à douze fois le montant de la somme blanchie et porter la peine de servitude pénale à 20 ans $^{94}$. le législateur soumet au paiement d'une amende en francs congolais pouvant aller de l'équivalent de 100.000 à 500.000 dollars américains, toute personne morale impliquée, de quelque manière que ce soit dans le financement d'activités terroristes, sans préjudice de la responsabilité pénale individuelle des dirigeants ou agents éventuellement impliqués ${ }^{95}$.

La personne coupable de financement du terrorisme subit, en outre, la confiscation des biens qui sont l'instrument ou le produit de l'infraction. Dans le cas de condamnation pour infraction de blanchiment consommée ou tentée, sera ordonnée la confiscation des biens objets de l'infraction, y compris les revenus et autres avantages qui en ont été tirés, à quelque personne qu'ils appartiennent, à moins que leur propriétaire n'établisse qu'il les a acquis en versant effectivement le juste prix ou en échange des prestations correspondant à leur valeur ou à tout autre titre licite et qu'il en ignorait l'origine illicite; mais aussi doivent être confisqués, des biens appartenant, directement ou indirectement, à une personne condamnée pour fait de blanchiment des capitaux ${ }^{96}$.

Par ailleurs, en cas d'infraction constatée par le tribunal, lorsqu'une condamnation ne peut être exécutée contre son ou ses auteurs, celui-ci peut néanmoins ordonner la confiscation des biens sur lesquels l'infraction a porté. Peut, en outre, être prononcée, la confiscation des biens du condamné à hauteur de l'enrichissement, réalisé par lui depuis la date du plus ancien des faits justifiant sa condamnation, à moins qu'il n'établisse l'absence de lien entre l'enrichissement et l'infraction. Lorsqu'il y a confusion des biens provenant directement ou indirectement de l'infraction et d'un bien acquis légitimement, la confiscation de ce bien n'est ordonnée qu'à concurrence de la valeur estimée par la juridiction du bien mal acquis $^{97}$.

\section{2) La répression du terrorisme}

Le législateur congolais n'a pas inscrit le terrorisme dans le code pénal ordinaire. Il a fallu attendre des législations spéciales pour enfin retrouver l'infraction de terrorisme y être reprise. Le terrorisme est définit dans le code pénal militaire à son article $157^{98}$ et dans la loi $\mathrm{n}^{\circ} 04 / 016$ du 19 juillet 2004 portant lutte contre le blanchiment de capitaux et financement du terrorisme. Le code pénal ordinaire qui date du 30 janvier 1940, époque à laquelle ce fléau n'avait pas encore assez d'ampleur sur le continent africain et ne captait pas autant

93 Art. 41 de la Loi nº4/016 du 19 juillet 2004, Ibidem.

94 Art. 39 de la Loi n $04 / 016$ du 19 juillet 2004, Ibidem.

95 Art. 42 de la Loi n ${ }^{\circ} 4 / 016$ du 19 juillet 2004, Op.cit.

96 Art. 47 de la Loi n04/016 du 19 juillet 2004, Op.cit.

97 Art. 47 de la Loi n04/016 du 19 juillet 2004, Idem.

98 Art. 157 de la Loi nº24/2002 du 18 novembre 2002 portant code pénal militaire, Journal Officiel de la RDC, nº spécial, 20 mars 2003. 
l'attention d'opinions publiques ni de la communauté internationale en générale, moins encore celle de la région et à certains auteurs de conclure qu'avant 1940 le terrorisme n'entrainait pas des massacres de grande ampleur en l'exception de quelque souvenir macabre notamment celui des terroristes sionistes, du Gang Stern, qui assassinèrent le ministre plénipotentiaire britannique en résidence au Moyen-Orient ${ }^{99}$.

Dans le cadre de la répression de ce fléau, les Conventions internationales joue un rôle important, surtout dans les cas où le législateur congolais reste moins explicites sur certaines notions qui exigeraient plus d'explications, cette lutte s'appuieraient utilement notamment à la Convention de l'OUA sur la Prévention et la Lutte contre le Terrorisme de 1999, à la Stratégie antiterroriste mondiale de l'Organisation des Nations Unies, New York de 2006, voire même à la Convention des Nations Unies contre la criminalité transnationale organisée 2001 .

\section{Conclusion}

La position géostratégique de la République Démocratique du Congo est d'une importance capitale et déterminante pour la paix, la sécurité et la stabilité de l'Afrique centrale, une région menacée en ces jours par l'expansion des groupes terroristes Al-Shabaab à l'Est et Boko haram, Etat islamique en Afrique de l'Ouest, vers le Nord-Ouest de la région. La situation est de plus en plus alarmante puisque nombreux pays africains, et surtout ceux de l'Afrique centrale, sont des terreaux festiles au terrorisme et donc, la dynamique de l'exportation de la menace est certaine.

La République Démocratique du Congo qui, disposant d'un cadre juridique pas du tout satisfaisant au regard des enjeux et exigences en présence et des institutions spécialisées nationales qui fonctionnent au milieu des textes imprecis et des pratiques sans consistances ni moyens nécesaires pour leurs mise en oeuvre, ne joue pas le rôle qu'il faut à la hauteur de sa grandeur et de son importance. Et pourtant les exigences de la redéfinition des politiques et stratégies de résilience face à ces fléaux impliquent des moyens matériels, stratégiques, intellectuels et humains énormes et spécifiques dont les Etats de la région ne semblent pas réunir, moins encore la République Démocratique du Congo, ouvrant ainsi la voie aux violations massives des droits de l'homme par des groupes criminels impénitents et constituant un danger imminent à l'existence de l'Etat de droit dans la région et en République Démocratique du Congo.

Il faut en définitive constaté que les activités criminelles des terroristes islamistes radicaux bousculent les dispositifs de défense et de sécurité des pays de la région, et les appellent au renouvellement de leurs doctrines en matière de prévention et de gestion des risques et menaces, dont les menaces terroristes. Mais, dans la mesure où la faillite de l'Etat s'avère générale et globalisante, la redéfinition de la place de la masse populaire aux côtés

99 KHALED AMAYREH, « avant l'Etat juif, trois organisations terroristes juives œuvraient en Palestine », Le huffington post, 9 février 2009, disponible sur http://archives-lepost.huffingtonpost.fr/ consulté le 21 aout 2015. 
de l'Etat dans cette lutte et l'identification des mesures prioritaires et appropriée à la région serait d'une importance capitale.

\section{Bibliographie}

\section{Textes juridiques}

CONFERENCE INTERNATIONALE SUR LA REGION DES GRANDS LACS, Paix et Sécurité, Projet sur la criminalité transnationale et le terrorisme, Fusion des Projets No. 1.3.1 et 1.3.2, Révision du 30 juin 2005.

Convention arabe relative à la répression du terrorisme, Caire, le 22 avril 1998. En vigueur depuis le 7 mai 1999.

Décret $\mathrm{n}^{\circ} 08 / 20$ du 24 septembre 2008 portant organisation et fonctionnement d'une Cellule nationale des renseignements financiers « CENAREF ».

Décret n ${ }^{\circ} 08 / 21$ du 24 septembre 2008 portant création du Comité consultatif de lutte contre le blanchiment des capitaux et financement du terrorisme, COLUB en sigle, Journal Officiel de la RDC, $n$ ${ }^{\circ} 20,15$ octobre 2008.

Décret $n^{\circ} 08 / 22$ du 24 septembre 2008 portant création du fonds de lutte contre le crime organisé, en sigle « FOLUCCO », Journal Officiel de la RDC, $n^{\circ} 20,15$ octobre 2008.

Loi n⿳024/2002 du 18 novembre 2002 portant code pénal militaire, Journal Officiel de la RDC, $n^{\circ}$ spécial, 20 mars 2003.

Loi n04/016 du 19 juillet 2004 Portant lutte contre le blanchiment des capitaux et financement du terrorisme.

\section{Doctrine}

\section{A. Ouvrages}

BAYART J. F., « Le crime transnational et la formation de l’État », Politique africaine $n^{\circ} 93$ - mars 2004, pp. 93-104.

BERNARD HUYGHE F., Anthologie de textes sur la polémologie, Une science de la guerre?, Paris, Gipri, 2008.

BIYOYA MAKUTU KAHANDJA P., La géopolitique de l'instabilité dans la région des Grands lacs. Réflexion sur les réfugiés, ces acteurs/auteurs des mutations géostratégiques, Kinshasa, L'harmat$\tan , 2009$.

CAMPANA A. et HERVOUET G., dir., Terrorisme et insurrection : évolution des dynamiques conflictuelles et réponses des Etats, Québec, Presses de l'Université du Québec, 2013.

CAMUS A., L'homme révolté, Paris, Gallimard, 1951.

CEDRONIO M., Hannah Arendt : Politique et histoire. La démocratie en danger, Paris, L'Harmattan, 1999.

CLEMESAC N., Comprendre le phénomène des enfants soldats, JRS, Bujumbura, février 2007.

COUTTE A., Irak. La ruée vers l'or, France, Mercuse, 2003. 
GUIBBAUD Pauline, BOKO HARAM Histoire d'un islamisme sahélien, Paris, L'Harmattan, 2015.

KIFWABALA TEKILAZAYA, FATAKI wa LUHINDI D., WETSH'OKONDA KOSO M., République démocratique du Congo. Le secteur de la justice et l'Etat de droit, Johannesburg, Open siciety foundation, 2013.

MBATA B. MANGU A., Abolition de la peine de mort et constitutionnalisme en Afrique, Paris, L'Harmattan, 2011.

NTUMBA-LUABA LUMU D.A., Droit constitutionnel général, Kinshasa, éditions universitaires africaines, 2007.

NZONGOLA-NTALAJA G., Faillite de la gouvernance et crise de la construction nationale au Congo-Kinshasa. Une analyse des luttes pour la démocratie et la souveraineté nationale, Kinshasa-Montréal-Washington, ICREDES, 2015.

B. Articles

BERGHEZAN G., Boko Haram, fiche documentaire, Note d'Analyse du GRIP, Bruxelles, 8 janvier 2016.

BIDA KOYAGBELE P., « Centrafrique : Boko haram et l'état islamique (EI) au cœur de l'Afrique », JNRD, 2015, pp. 8-9.

BRAECKMAN C., « Kivu: des recrutements qui posent question », Le carnet de Colette Braeckman, mars 2015.

CERDA-GUZMAN C., « La Constitution : une arme efficace dans le cadre de la lutte contre le terrorisme? », Revue française de droit constitutionnel, 2008/1 (n 73), p. 41-63.

DEVLIN-FOLTZ Z., « Les États fragiles de l'Afrique : vecteurs de l'extrémisme, exportateurs du terrorisme », Bulletin de la sécurité africaine, $n^{\circ}$ 6, Washington DC, août 2010.

DIDIMALA LODGE, « Les Etats fragiles en Afrique: un paradigme utile pour l'action?, Rapport de conférence ", Observatoire de l'Afrique, Aout 2008.

EBA'A ATYI R. et BAYOL N., « Les forets de la République démocratique du Congo en 2008 », observatoire-comifac, $n^{\circ} 07$, juillet 2008, pp. 115-128.

EOUZAN J.P., « Déplacements de populations et trypanosomiase humaine en Afrique centrale », International Journal of Tropical Insect Science, Vol.1, 19 September 2011.

GAULME F., « Les économies de guerre, nouveau mal du siècle », Études 2005/11 (Tome 403), p. 441-452.

HASSAN DJAMA FARAH, « Djibouti : un hub anti-piraterie et un acteur principal de la médiation dans la crise somalienne », Diplomatie, un autre regard sur le monde, $n^{\circ} 56$ - Mai-juin 2012, pp. 3-5.

JIHAD GILLON et MARBOT O., « Tout sur 1'Etat islamique. Son histoire-Ses chefs-Ses ressourcesSes objectifs ", La Revue pour l'intelligence du monde, Bimestriel, n ${ }^{\circ} 55-56$, septembre-octobre 2015, pp. 18-37. 
NDESHYO RURIHOSE O. et ABDOU MUHIMA M., " La criminalité transnationale organisée en Afrique : une grave menace contre la paix et la sécurité des africains ", Revue internationale de criminologie et de police technique et scientifique, Vol. LXVIII, $n^{\circ} 2$, Genève, Avril-Juin 2015, pp. 131-157.

NYABIRUNGU mwene SONGA, « Le blanchiment de capitaux et le financement du terrorisme. Exposé au Séminaire de fin-Consult du 29 au 30 avril 2009 », Annales de la Faculté de Droit de l'Université de Kinshasa, Kinshasa, Editions Droit et Société « DES », Juin 2014, pp. 404-447.

NZONGOLA-NTALAJA G., "The fragile State and crisis in eastern Congo », African Journal of democraty and governance (AJDG), Vol. 2, $n^{\circ} 1 \& 2$, Kinshasa, 2015, pp. 15-37.

OGOULAT A-D., « Afrique Centrale et Golfe de Guinée : Géopolitique des Termes de l'Echange entre deux Regionymes Sous-contentieux », Enjeux, $n^{\circ}$ 26, 2006.

RIGAUD C., « RDC: des djihadistes au Kivu? », Afrikarabia, mars 2015.

RIVERO J., «L'Etat moderne peut-il être encore un Etat de droit? », Cairn, 2003, pp. 65-101.

SADIA SULAIMAN, " Pakistan: les nouveaux talibans entre deux feux », Alternatives Internationales $n^{\circ}$ 041, décembre 2008.

SAURAL A., «Les élections au Nigeria perturbées par Boko Haram », Egalité et réconciliation, 28 mars 2015.

VEREYCKER Karel, «HSBC, « voyage au cœur de la finance criminelle », Solidarité et progrès, mars 2015.

\section{Rapports}

Conseil de sécurité, Rapport du Secrétaire général sur la situation en Afrique centrale et les activités du Bureau régional des Nations Unies pour l'Afrique centrale, 7461e séance, le 11 juin 2015.

PSC/AHG/2.(CDLV), Rapport de la président de la commission de l'Union africaine sur le terrorisme et l'extrémisme violent en Afrique, 455ème réunion, au niveau des Chefs d'Etat et de gouvernement, Nairobi, 2 septembre 2014.

UNICEF, Les enfants soldats, Rapport 2012,

\section{Internet}

DIFFALAH Sarah, "Les shebab ne sont pas qu'un groupe terroriste, Politique africaine, disponible sur http://tempsreel.nouvelobs.com/, consulté le 21 aout 2015.

Global Terrorism Databased: GTD Variables \& Inclusion Criteria (Base de données du terrorisme mondial : variables et critères d'inclusion dans la GDT). National Consortium for the Study of Terrorism and Responses to Terrorism (START), mai 2015; http://www.start.umd.edu/gtd/downloads/Co debook.pdf, consulté le 26 novembre 2015.

GOMEZ M., «La banque HSBC finançait le terrorisme », disponible sur www.http://magoturf.over-bl og.com., consulté le 21 aout 2015. 
KIBANGULA T., « RDC - Julien Paluku : Les islamistes Shebaab combattent aux côtés du M23 et de l'ADF-Nalu », Jeuneafrique, 16 juillet 2013, disponible sur http://www.jeuneafrique.com, consulté le 5 février 2014.

LUMUMBA R., RDC / Shebaab ou Boko Haram: Une nouvelles base arrière des islamistes à l'Est du Congo avec des recrutements tout azimut dans les mosquées et lieu de prière Musulman, disponible sur http://www.alterinfo.net, consulté le 21 aout 2015.

UN, The List established and maintained by the Al-Qaida Sanctions Committee with respect to individuals, groups, undertakings and other entities associated with Al-Qaida, Last updated on $26 \mathrm{Au}-$ gust 2014, disponible sur le cite http://www.un.org/sc/committees/1267/delisting.shtml., consulté le 02 septembre 2014

ZENN J., «Leadership Analysis of Boko Haram and Ansaru in Nigeria », CTC Sentinel, vol.7, $n^{\circ}$, février 2014, disponible sur le cite https://www.ctc.usma.edu/wp-content/uploads/2014/02/CTCSe ntinel-Vol7Iss2.pdf., consulté le 20 mai 2014. 